\title{
Predictive Significance of Ki-67 and Platelet Lymphocyte Ratio in Patients with Gastric Cancer Receiving Neoadjuvant FLOT Chemotherapy
}

\author{
Hatice Yilmaz ${ }^{1}$, Guzin Demirag ${ }^{1}$, Yurdanur Sullu ${ }^{2}$ and Ali Yilmaz ${ }^{3}$ \\ ${ }^{1} 1$ Department of Medical Oncology, Ondokuz Mayıs University Faculty of Medicine, Samsun, Turkey \\ ${ }^{2}$ Department of Pathology, Ondokuz Mayıs University Faculty of Medicine, Samsun, Turkey \\ ${ }^{3}$ Department of Medical Oncology, Atatürk University Faculty of Medicine, Erzurum, Turkey
}

\begin{abstract}
Objective: We aimed to determine the predictive significance of Ki-67 and platelet lymphocyte ratio (PLR) in patients with gastric cancer (GC), who received fluorouracil, leucovorin, oxaliplatin, docetaxel (FLOT) as neoadjuvant chemotherapy (NAC).

Study Design: Descriptive study.

Place and Duration of Study: Department of Medical Oncology, Faculty of Medicine, Ondokuz Mayıs University, Samsun, Turkey from March 2016 to January 2020.

Methodology: Seventy-five patients with GC, who received FLOT treatment as NAC were included in the study. Ki-67 and PLR, which were examined pre-NAC and aft-NAC, were recorded. Associations between clinical-histopathological parameters with disease-free survival (DFS) and overall survival (OS) were analysed using Kaplan-Meier curves. Cox-regression analysis was used to assess their prognostic values.

Results: There was a statistically significant difference between pre-NAC and aft-NAC Ki-67, and aft-NAC PLR values between groups with complete response, partial response, and stable disease aft-NAC ( $p: 0.023, p:<0.001$; and $p: 0.001$, respectively). When the patients were grouped according to the pre-NAC and aft-NAC Ki-67 changes, a significant difference was found in terms of OS ( $p<0.001)$. High pre-NAC and high aft-NAC Ki-67 were associated with shorter DFS and OS (p: 0.042, p: 0.049; p: 0.027, and p: 0.001, respectively). The high preNAC PLR was associated with shorter OS, while the high aft-NAC PLR was associated with shorter DFS ( $p: 0.018$, and p: 0.001, respectively). In multivariate analysis, aft-NAC Ki-67 was found to be an independent prognostic factor for OS.

Conclusion: Ki-67 and PLR have predictive significance in GC patients treated with neoadjuvant FLOT. Ki-67 is an independent prognostic marker for OS in gastric cancer.
\end{abstract}

Key Words: FLOT, Gastric cancer, Ki-67, Platelet lymphocyte ratio.

How to cite this article: Yilmaz H, Demirag G, Sullu Y, Yilmaz A. Predictive Significance of Ki-67 and Platelet Lymphocyte Ratio in Patients with Gastric Cancer Receiving Neoadjuvant FLOT Chemotherapy. J Coll Physicians Surg Pak 2021; 31(05):546-552.

\section{INTRODUCTION}

Gastric cancer (GC) is the 5th most common cancer and the third most common cause of cancer-related deaths. ${ }^{1}$ Since, approximately $2 / 3$ of the patients are in the locally advanced stage at the time of diagnosis, they are generally not suitable for surgical treatment, and neoadjuvant chemotherapy (NAC) is a more suitable treatment option for these patients. ${ }^{1}$ Recent studies have shown that for patients with stage II-III disease, the use of fluorouracil, leucovorin, oxaliplatin and docetaxel (FLOT) therapy as NAC regimen is more effective than other chemotherapy regimens. ${ }^{2}$ Evaluation of NAC effectiveness is essential to decide on the selection of adjuvant therapy.

Correspondence to: Dr. Hatice Yllmaz, Department of Medical Oncology, Faculty of Medicine, Ondokuz Mayıs University, Samsun, Turkey

E-mail: drhaticeyilmaz19@gmail.com

Received: January 30, 2021; Revised: March 20, 2021;

Accepted: April 09, 2021

DOI: https://doi.org/10.29271/jcpsp.2021.05.546
In addition to radiological evaluation based on Response Evaluation Criteria In Solid Tumours (RECIST) criteria and pathological response ratings such as Mandard score have been used in efficacy evaluation. The role of some different biomarkers in evaluating efficacy has also been recently investigated. ${ }^{3}$ One of these markers, Ki-67, is a nuclear protein which is commonly used to demonstrate cellular proliferation detected in the cell cycle G1, S, G2, and M phases; but not detected in the resting phase of $\mathrm{G} 0$, and is also associated with tumourcell proliferation rate. ${ }^{4}$ Although some studies in patients with locally advanced GC have shown a correlation between radiological imaging and histopathological findings, and high $\mathrm{Ki}-67$ is associated with poor prognosis, the importance of Ki-67 expression as a NAC predictor in GC patients is still uncertain. ${ }^{5}$ Apart from proliferative markers such as Ki-67, systemic inflammatory response indicators such as lymphocyte, neutrophil, and platelet are also associated with the development and progression of many tumours. Tumour cells can alter the balance of pro-inflammatory mediators by increasing neutrophil, monocyte, and platelet count in peripheral blood and decreasing lymphocyte count. ${ }^{6}$ The platelets play an important role in tumour develop- 
ment and metastasis, although the mechanism of this action is still unknown, the increased platelet count is associated with shorter survival in many malignancies. ${ }^{7}$ Lymphocytes induce cytotoxic cell death and inhibit the proliferation and migration of tumour cells. ${ }^{8}$ Inflammatory markers such as PLR, obtained from systemic inflammatory response indicators, are associated with prognosis in many cancers such as GC, colon, and prostate. ${ }^{9-11}$ However, the role of these inflammatory markers in response to treatment in patients with GC is not yet clear.

Therefore, the aim of this study was to investigate the predictive significance of Ki-67 and PLR levels before (pre-NAC) and after (aft-NAC) NAC in terms of response assessment to the FLOT regimen in patients with $\mathrm{GC}$. It was also aimed to show the importance of pre-NAC and aft-NAC level changes of these markers for the determination of tumour chemosensitivity.

\section{METHODOLOGY}

Seventy-five patients with gastric adenocarcinoma, who underwent FLOT treatment as an NAC regimen in the Medical Oncology Department between 2016-2020 were evaluated retrospectively.

The inclusion criteria were age 18-75 years; gastric adenocarcinoma confirmed via gastroscopic biopsy; no distant organ metastasis; good performance status (0-2) of Eastern Clinical Oncology Group (ECOG), and good haematological, hepatic, renal and cardiac function. Exclusion criteria included another malignancy, systemic infection, haematological and autoimmune diseases.

Clinical variables such as gender, age, ECOG performance status, tumour location, tumour size, lymph node involvement, tumour stage, and laboratory data such as pre-NAC lymphocyte and platelets were obtained from archive files. Biopsy findings at the time of pre-NAC diagnosis and pathological findings in the aft-NAC operation material such as Ki-67, histological type, Barrett epithelium, perineural invasion (PNI), lymphovascular invasion (LVI) were taken from the Pathology Department electronic registry system. In the immunohistochemical study with a primary antibody, Ki-67 expression was determined by selecting the areas with the most intense staining (hot-spot) in the light microscope and calculating the percentage of Ki-67 stained tumour cells. PLR was calculated by dividing platelet count by lymphocyte count. Pre-NAC and four cycles of aft-NAC PLR values were recorded. Aft-NAC response evaluation was performed by grouping as complete response, partial response, stable, and progressive disease according to RECIST criteria.

The Ki-67 and PLR were dichotomised based on a receiver operating characteristic (ROC) curve analysis. The best cut-off values were determined for pre-NAC / aft-NAC Ki-67 $(60,30)$ and PLR $(336.7,110)$. According to the ROC analysis, area under curve (AUC) values for pre-NAC Ki-67 (0.646), aft-NAC Ki-67 (0.682), pre-NAC PLR (0.50), and aft-NAC PLR (0.594) werefound asindicated.
Table I: Clinical and demographic characteristics of gastric cancer patients treated with neoadjuvant FLOT.

\begin{tabular}{|c|c|}
\hline & n (\%) \\
\hline \multicolumn{2}{|l|}{ Gender } \\
\hline Female & $27(36.0)$ \\
\hline Male & $48(64.0)$ \\
\hline \multicolumn{2}{|l|}{ Age (year) } \\
\hline$<65$ & $45(60.0)$ \\
\hline$\geq 65$ & $30(40.0)$ \\
\hline \multicolumn{2}{|l|}{ ECOG } \\
\hline $0-1$ & $43(57.3)$ \\
\hline 2 & $32(42.7)$ \\
\hline \multicolumn{2}{|l|}{ Tumor localisation } \\
\hline Cardia & $30(40.0)$ \\
\hline Corpus & $24(32.0)$ \\
\hline Antrum & $21(28.0)$ \\
\hline \multicolumn{2}{|l|}{ Barrett mucosa } \\
\hline Yes & $8(10.7)$ \\
\hline No & $67(89.3)$ \\
\hline \multicolumn{2}{|c|}{ Lauren classification } \\
\hline Diffuse & $20(26.7)$ \\
\hline Intestinal-mix & $54(72.0)$ \\
\hline Unknown & $1(1.3)$ \\
\hline \multicolumn{2}{|l|}{ Grade } \\
\hline 1 & $5(6.7)$ \\
\hline 2 & $54(72.0)$ \\
\hline 3 & $16(21.3)$ \\
\hline \multicolumn{2}{|l|}{ Stage group } \\
\hline 2 & $18(24.0)$ \\
\hline 3 & $57(76.0)$ \\
\hline \multicolumn{2}{|c|}{ Response to neoadjuvant treatment } \\
\hline Complete response & $26(34.7)$ \\
\hline Partial response & $26(34.7)$ \\
\hline Stable disease & $23(30.7)$ \\
\hline \multicolumn{2}{|c|}{ Type of gastrectomy } \\
\hline Subtotal & $27(36.0)$ \\
\hline Total & $48(64.0)$ \\
\hline \multicolumn{2}{|l|}{ Type of resection } \\
\hline RO & $64(85.3)$ \\
\hline R1 & $11(14.7)$ \\
\hline \multicolumn{2}{|c|}{ Lymphovascular invasion } \\
\hline No & $42(56.0)$ \\
\hline Yes & $33(44.0)$ \\
\hline \multicolumn{2}{|l|}{ Perineural Invasion } \\
\hline No & $52(69.3)$ \\
\hline Yes & $23(30.7)$ \\
\hline \multicolumn{2}{|l|}{ Pathological stage } \\
\hline 1 & $17(22.7)$ \\
\hline 2 & $43(57.3)$ \\
\hline 3 & $15(20.0)$ \\
\hline \multicolumn{2}{|l|}{ Status } \\
\hline Alive & $67(89.3)$ \\
\hline Death & $8(10.7)$ \\
\hline
\end{tabular}

According to NAC's response, the groups' relationships with the pre-NAC and aft-NAC Ki-67 and PLR values and the differences in the OS time according to the aft-NAC alteration groups of these parameters were tested with One-Way ANOVA. Tukey's post-hoc tests were used for subgroup analyses. Overall survival (OS) was calculated from the date of diagnosis to death and censored at the date of the last follow-up for survivors. Disease-free survival (DFS) was calculated from the date of surgery to the date of relapse or death and censored at the date of the last follow-up for survivors without recurrence. 
Table II: Survival of patients before and after NAC, according to Ki-67 level change.

\begin{tabular}{|c|c|c|c|c|c|c|}
\hline Change in Ki-67 with chemotherapy & $\mathbf{n}$ & OS mean \pm SD & Change in Ki67 with chemotherapy & $\mathbf{n}$ & OS mean \pm SD & $\mathbf{p}$ \\
\hline \multirow[t]{3}{*}{ Pre-NAC Ki67 $7^{\text {low }}$-aft NAC Ki $67^{\text {low }}$} & 26 & $28.69 \pm 6.4$ & Pre-NAC Ki $67^{\text {low }}$-aft NAC Ki $67^{\text {hi }}$ & 10 & $22.30 \pm 2.0$ & 0.006 \\
\hline & & & Pre-NAC Ki $67^{\text {hi }}$-aft NAC Ki $67^{\text {low }}$ & 26 & $28.15 \pm 5.2$ & 0.980 \\
\hline & & & Pre-NAC Ki67 $7^{\text {hi }}$-aft NAC Ki $67^{\text {hi }}$ & 13 & $22.0 \pm 2.1$ & 0.001 \\
\hline \multirow[t]{2}{*}{ Pre-NAC Ki67 $7^{\text {low }}$-aft NAC Ki $67^{\text {hi }}$} & 10 & $22.30 \pm 2.0$ & Pre-NAC Ki $67^{\text {hi }}$-aft NAC Ki $67^{\text {low }}$ & 26 & $28.15 \pm 5.2$ & 0.014 \\
\hline & & & Pre-NAC Ki $67^{\text {hi }}$-aft NAC Ki $67^{\text {hi }}$ & 13 & $22.0 \pm 2.1$ & 0.999 \\
\hline Pre-NAC Ki $67^{\text {hi }}$-aft NAC Ki $67^{\text {low }}$ & 26 & $28.15 \pm 5.2$ & Pre-NAC Ki67 $7^{\text {hi }}$-aft NAC Ki $67^{\text {hi }}$ & 13 & $22.0 \pm 2.1$ & 0.003 \\
\hline
\end{tabular}

Table III: Univariate and multivariate analysis of parameters for DFS and OS.

\begin{tabular}{|c|c|c|c|c|c|c|c|c|}
\hline & Univariate DFS HR & $\mathbf{p}$ & Multivariate DFS HR & $\mathbf{p}$ & Univariate OS HR & $\mathbf{p}$ & Multivariate OS HR & $\mathbf{p}$ \\
\hline Gender & $0.055(0.012-0.247)$ & $<0.001$ & $0.038(0.003-0.555)$ & 0.017 & $0.189(0.038-0.942)$ & 0.042 & $0.318(0.054-1.866)$ & 0.205 \\
\hline Age & $0.721(0.283-1.834)$ & 0.492 & & & $0.430(0.087-2.136)$ & 0.302 & & \\
\hline ECOG & $1.707(0.649-4.492)$ & 0.279 & & & $8.581(1.056-69.762)$ & 0.044 & 7.988(0.974-65.494) & 0.053 \\
\hline $\begin{array}{l}\text { Tumor } \\
\text { localization }\end{array}$ & $1.172(0.817-1.680)$ & 0.388 & & & $1.765(0.893-3.491)$ & 0.102 & & \\
\hline Barrett mucosa & $0.405(0.145-1.130)$ & 0.084 & & & $0.403(0.081-1.999)$ & 0.266 & & \\
\hline $\begin{array}{l}\text { Lauren } \\
\text { classification }\end{array}$ & $3.133(1.209-8.121)$ & 0.019 & $3.443(0.72416 .373)$ & 0.120 & $4.362(0.783-24.294)$ & 0.093 & & \\
\hline Grade & $0.679(0.241-1.912)$ & 0.463 & & & $1.532(0.408-5.754)$ & 0.527 & & \\
\hline $\begin{array}{l}\text { AJCC TNM } \\
\text { Stage }\end{array}$ & $0.660(0.251-1.739)$ & 0.401 & & & $0.313(0.078-1.252)$ & 0.101 & & \\
\hline $\begin{array}{l}\text { Response to } \\
\text { neoadjuvant } \\
\text { treatment }\end{array}$ & $2.413(1.291-4.511)$ & 0.006 & $1.347(0.606-2.992)$ & 0.465 & $0.879(0.374-2.062)$ & 0.766 & & \\
\hline $\begin{array}{l}\text { Type of } \\
\text { gastrectomy }\end{array}$ & $0.268(0.104-0.691)$ & 0.006 & $0.634(0.151-2.661)$ & 0.534 & $0.315(0.075-1.321)$ & 0.114 & & \\
\hline $\begin{array}{l}\text { Type of } \\
\text { resection }\end{array}$ & $3.236(1.297-8.073)$ & 0.012 & $6.025(1.368-26.539)$ & 0.018 & $0.712(0.088-5.791)$ & 0.751 & & \\
\hline LVi & $7.331(2.098-25.620)$ & 0.002 & $0.451(0.000-1.669)$ & 0.937 & $1.216(0.304-4.870)$ & 0.782 & & \\
\hline PNI & $6.465(1.869-22.365)$ & 0.003 & $0.570(0.000-2.028)$ & 0.955 & $2.009(0.501-8.064)$ & 0.325 & & \\
\hline $\begin{array}{l}\text { Pathological } \\
\text { Stage }\end{array}$ & $4.185(1.830-9.569)$ & 0.001 & $0.944(0.297-2.996)$ & 0.922 & $2.419(0.796-7.353)$ & 0.119 & & \\
\hline Pre-NAC Kí7 & $2.402(0.911-6.335)$ & 0.077 & & & $6.345(0.780-51.613)$ & 0.084 & & \\
\hline Aft-NAC Ki67 & $2.459(0.984-6.144)$ & 0.054 & & & $8.260(1.627-41.928)$ & 0.011 & $7.582(1.329-43.251)$ & 0.023 \\
\hline Pre-NAC PLR & $2.513(0.577-10.951)$ & 0.220 & & & $5.549(1.116-27.593)$ & 0.036 & $2.524(0.427-14.912)$ & 0.307 \\
\hline Aft-NAC PLR & $\begin{array}{l}10.765(1.436 \\
80.708)\end{array}$ & 0.021 & $2.827(0.231-34.660)$ & 0.417 & $2.979(0.366-24.215)$ & 0.307 & & \\
\hline
\end{tabular}

Associations between clinical and histopathological parameters with OS and DFS were analysed using KaplanMeier curves and compared by the log-rank test.

Univariate and multivariate cox-regression analyses were performed to determine the effects of potential prognostic factors, including gender, age, ECOG, site, Barrett epithelium, Lauren classification, grade, American Joint Committee on Cancer (AJCC) TNM stage and response to neoadjuvant therapy for DFS and OS. Hazard ratios (HRs) were estimated from the Cox analysis and were reported as relative risks with corresponding $95 \%$ confidence intervals (Cls). Categorical data were expressed as frequencies and percentages, while continuous data as mean \pm S.D. All analyses were performed using the SPSS statistical software package (SPSS statistics version 21.0). A p <0.05 was considered statistically significant.

\section{RESULTS}

Out of the 75 patients included in this study, 27 (36\%) were females and $48(64 \%)$ were males. The mean age of the patients was $61.93 \pm 7.9$ years. The clinical and demographic characteristics of the patients are in Table I. After FLOT treatment as an NAC regimen, 26 (34.7\%) patients had complete response, 26 (34.7\%) patients had partial response, and $23(30.7 \%)$ patients had stable disease. At the end of $26.49 \pm 5.7$ months (14-36 months) with a mean follow-up period, 19 (25.3\%) patients developed progression, while $8(10.7 \%)$ patients died. 
Mean Ki-67 and PLR values pre-NAC and aft-NAC in all patient groups were $60.77 \pm 26.5,28.53 \pm 24.3,142.76 \pm$ 72.3 , and $179.36 \pm 95.2$, respectively. In terms of response to NAC, pre-NAC Ki-67 was $55.92 \pm 27.6$ in the fully responsive group, $54.58 \pm 28.2$ in the partial responsive group, and $73.26 \pm 19.0$ in the stable disease group. AftNAC Ki-67 was $13.46 \pm 10.8$ in the fully responsive group, $27.69 \pm 20.2$ in the partial responsive group, $46.52 \pm 28.1$ in the stable disease group, and the difference between the groups were significant ( $p$ : 0.023, and $p<0.001$, respectively). The subgroup analysis showed that there was no statistically significant difference between the complete response and stable response groups in the pre-NAC Ki-67 and the complete response group and the partial response group, while there was a significant difference between the partial response and stable response groups, ( $p: 0.052$, and $p$ : 0.98, and p: 0.033, respectively).

The Ki-67 in the aft-NAC period was found to be significantly different between the groups of complete response and partial response, complete response and stable response, partial response, and stable response ( $p$ : 0.039, $p<0.001$, and p: 0.006, respectively).

In terms of NAC response, pre-NAC PLR was $138.28 \pm 89.6$ in the complete responsive group, $146.98 \pm 61.8$ in the partial responsive group, and $143.04 \pm 63.83$ in the stable disease group, and no statistically significant difference was found (p:0.912). Aft-NAC PLR was $159.72 \pm 75.8$ in the complete responsive group, $147.14 \pm 65.7$ in the partial responsive group, and $237.99 \pm 117.4$ in the stable disease group, and there was a statistically significant difference ( p: 0.001). When subgroup analysis was performed, there was no difference between a complete response and partial response groups in aft-NAC PLR, whereas there was a statistically significant difference between the groups of complete response and stable response, and partial response and stable response ( $p: 0.893, p: 0.007$, and $p$ : 0.025 , respectively).

When all patients were grouped according to pre-NAC and aft-NAC Ki-67 level change, there was a significant OS difference between the groups with $\mathrm{Ki}-67$ expression pre$\mathrm{NAC}^{\text {low }} \rightarrow$ aft-NAC low , pre-NAC ${ }^{\text {low }} \rightarrow$ aft-NAC ${ }^{\text {hi }}$, pre-NAC ${ }^{\text {hi }} \rightarrow$ aft$N A C^{\text {low }}$, pre-NAC ${ }^{\text {hi }} \rightarrow$ aft-NAC ${ }^{\text {hi }}(p<0.001)$. Subgroup analysis is shown in Table II. When patients are grouped according to pre-NAC and aft-NAC PLR change, OS \pm SD duration was $28.05 \pm 6.7$ months in the group with pre-NAC low $\rightarrow$ aft-NAC ${ }^{\text {low }}$ $\mathrm{PLR}, 25.84 \pm 5.3$ months in the group with pre-NAC ${ }^{\text {low }} \rightarrow$ aft$\mathrm{NAC}^{\text {hi }}$, and $26.00 \pm 4.6$ months in the group with pre-NAC ${ }^{\text {hi }} \rightarrow$ aft-NAC ${ }^{\text {hi }}$, and no statistically significant difference was found (p: 0.324).

As of the period of analysis, the average DFS was 30 (27.91-32.0) months and the OS was 34.13 (32.9-35.3) months. The high Ki-67 in both pre-NAC and aft-NAC periods was statistically significant for shorter DFS and OS ( $p: 0.042$, p: 0.027 ; p: 0.049 , and p: 0.001 , respectively). While high pre-NAC PLR was associated with shorter OS, high PLR in the aft-NAC period was statistically significant for shorter DFS ( $p$ : 0.018 , and p: 0.001, respectively).

A Cox proportional hazards model was used to evaluate the potential predictors as seen in Table III. In multivariate analysis, gender (HR: 0.038, 95\% C.I: 0.003-0.555 p:0.017), and type of resection (HR: 6.025, 95\% C.I: 1.368-26.539, p:0.018) were identified as independent prognostic factors for DFS. Aft-NAC Ki-67 (HR: 7.582, 95\% C.I: 1.329-43.251, p:0.023) was identified as independent prognostic factor for os (Table III).

\section{DISCUSSION}

Although GC is often diagnosed at a locally advanced stage, advances in multimodal therapy in recent years have significantly increased the life span and quality of life for these patients. ${ }^{12}$ It was also found aft-NAC Ki-67 as independent prognostic factors for OS in this study, where the authors investigated the predictive importance of Ki-67 and PLR in patients with GC, who received neoadjuvant FLOT therapy for the first time in the literature. In terms of response to NAC, the decrease in $\mathrm{Ki}-67$ was the highest in the complete responsive group, while it was the lowest in the group with stable disease.

Ki-67 protein is a cellular marker used to evaluate the proliferative activity of cancer cells, and a high Ki-67 level is associated with a poor prognosis of high grade and advanced-stage cancers. ${ }^{13}$ Although Ki-67 is associated with prognosis in many solid organ malignancies such as breast cancer and colorectal cancer, the prognostic role of $\mathrm{Ki}-67$ is still unclear in patients with GC., . $^{4,15}$

However, there is growing evidence that immuneinflammatory status plays an important role in carcinogenesis pathways in some cancers, including GC. Platelets, as one of the systemic inflammatory response indicators, mediate the migratory and angiogenetic effects of cancer cells in the tumoural microenvironment, on the contrary, lymphocytes show anti-tumoural activity. Therefore, PLR, which shows the balance between platelet count and lymphocyte count, may reflect the inflammatory state of the host. Recent studies have shown that PLR is prognostic in various cancers, including stomach cancer, colorectal cancer, ovarian clear cell carcinoma, and prostate cancer. ${ }^{6,10,11}$ Similarly, high PLR is associated with tumour aggressiveness in some malignancies, including GC. ${ }^{16-18}$

In a study by Sun et al., 58 advanced GC patients were included in the study, and the FOLFOX4 (5-fluorouracil / 
leucovorin combined and oxaliplatin) regimen was given as a neoadjuvant to 23 patients and as an adjuvant to 35 patients. The positive expression rate of $\mathrm{Ki}-67$ was lower in the group receiving NAC than in the adjuvant group. In addition, there was a negative correlation between the high levels of the Ki-67 and response to NAC. In the same study, although longer OS and PFS were reported in Ki-67 negative patients who received NAC than $\mathrm{Ki}-67$ positive patients, the difference was statistically significant for OS, but not significant for PFS. No significant relationship was found between Ki-67 expression and other variables such as invasion depth, nodal involvement, and TNM stage. ${ }^{19}$ In another study involving 167 patients by $\mathrm{Wu}$ et al. eighty-four of the patients were administered mFOLFOX7 (fluorouracil, leucovorin, oxaliplatin) as NAC and 83 of them as adjuvants.

Patients with better pathological responses had a higher Apoptosis index (Al), lower $\mathrm{Ki}-67$ index $(\mathrm{KI})$, and higher $\mathrm{Al} / \mathrm{KI}$ ratio, but $\mathrm{KI}$ and $\mathrm{Al} / \mathrm{KI}$ ratio were not significantly associated with prognosis. In this study, it was shown that $\mathrm{Al}$ and $\mathrm{KI}$ predicted NAC activity, and less differentiation and less vascular invasion were detected in the group where NAC was applied. Tumour invasion depth showed a significant correlation with $\mathrm{Al}$ in both groups, but only in the group receiving $N A C$, it was found to be significantly associated with $\mathrm{KI}^{20} \mathrm{Qu}$ et al. investigated the predictors for response to mFOLFOX7 given as NAC in 53 patients with Stage II-III GC, and found no significant difference in the levels of pre-NAC and aft-NAC tumour markers. In terms of NAC efficacy and protein expression, no significant difference was found for $\mathrm{Ki}-67 .{ }^{21}$ Unlike the two studies conducted by Sun et al. and Wu et al, this study included only the patients who received NAC, and evaluated pre-NAC and aft-NAC Ki-67 values. In terms of response to NAC, similar to these studies, in the pre-NAC and aft-NAC period, Ki-67 was the lowest in the complete response group, while it was the highest in the stable disease group; and the difference between the groups was statistically significant. Additionally, unlike other studies, aft-NAC Ki-67 were found prognostic for OS in this study. Unlike previous studies, all patients were grouped according to pre-NAC and aft-NAC Ki-67 level changes. The authors recorded a significant OS difference between the groups with $\mathrm{Ki}-67$ expression pre-NAC low $\rightarrow$ aft-NAC ${ }^{\text {low }}$, pre$\mathrm{NAC}^{\text {low }} \rightarrow$ aft-NAC ${ }^{\text {hi }}$, pre-NAC $\rightarrow$ aft-NAC ${ }^{\text {low }}$, pre-NAC pri $^{\text {hi }} \rightarrow$ aftNAC ${ }^{\text {hi }}$. Similarly, the Ki-67 level observed in the pre-NAC and aft-NAC period was associated with survival. Unlike the study of Qu et al; in this study, there was a significant decrease in the Ki-67 level after treatment, and the decrease in Ki-67 levels was significant in patients with good response to NAC. Unlike the studies by Wu et al. and Qu et al, it was observed the aft-NAC Ki-67 level as an independent prognostic factor.

Many studies showed that PLR is a useful biomarker to predict the prognostic outcome in GC patients. ${ }^{22,23}$ In a study of 91 patients diagnosed with stage II and III GC, the cut-off value for PLR before NAC [SOX (S-1 plus oxaliplatin) in 35 patients, XELOX (oxaliplatin and capecitabine) in 56 patients] was 162, and PLR was an independent prognostic factor for DFS and OS. It was found that the average DFS and OS durations in patients with low PLR were longer than patients with high PLR. In groups with low and high PLR, there was no significant difference in response to treatment. ${ }^{24}$ Gong et al. showed that patients with pre-NAC high PLR had worse OS than those with low PLR and found pre-NAC PLR as an independent prognostic factor for OS. In the same study, when patients were grouped according to pre-NAC and aft-NAC PLR changes, the duration of OS was statistically significantly longer in the group with pre-NAC PLR $^{\text {low }} \rightarrow$ aft-NAC PLR ${ }^{\text {low }}$ compared to the group with pre-NAC PLR $^{\text {low }} \rightarrow$ aft-NAC PLR ${ }^{\text {hi }}$, and in the group with pre-NAC PLR ${ }^{\text {hi }} \rightarrow$ aft-NAC PLR ${ }^{\text {low }}$ than the group with pre-NAC PLR ${ }^{\text {hi }} \rightarrow$ aft-NAC $P L R^{\text {hi }}$. OS duration was shorter in the group with pre-NAC PLR $^{\text {low }} \rightarrow$ aft-NAC PLR ${ }^{\text {hi }}$ compared to the group with pre-NAC $\mathrm{PLR}^{\text {hi }} \rightarrow$ aft-NAC PLR ${ }^{\text {low } 25}$ In this study, similar to these previous studies, pre-NAC high PLR was associated with worse OS. Consistent with the literature, high PLR in the aftNAC period was also significantly associated with shorter DFS. Unlike the study conducted by Gong et al. when the patients were grouped according to the pre-NAC and aft-NAC PLR changes, there was no statistically significant difference in OS time. However, in terms of response to NAC, the PLR in the aft-NAC period was lower in the groups with a complete response and partial response compared to the group with stable disease, and the difference between them was statistically significant.

Finally, this study is important because it is the first study to demonstrate that Ki-67 expression and PLR were associated with response to NAC in patients with $\mathrm{GC}$ receiving neoadjuvant FLOT therapy. However, this study has limitations as it was retrospective and included a limited number of patients. Therefore, we think that future prospective studies involving more patients are needed.

\section{CONCLUSION}

In GC patients treated with FLOT as the NAC regimen, the Ki-67 expression and PLR might be determinants of efficacy of treatment. Therefore, a routine evaluation of Ki-67, one of the immunohistochemical findings, and PLR, one of the haematological markers, may provide an advantage to determine the treatment strategy in patients with GC.

\section{ETHICAL APPROVAL:}

All procedures performed in this study involving human participants were in accordance with the ethical standards of the Institutional Research Committee, and with the 1964 Helsinki Declaration and its later amendments or comparable ethical standards. This study was approved by Ondokuz Mayıs University Medical Ethics Committee (2019/327). 


\section{PATIENTS' CONSENT:}

Since it was designed as a retrospective study, the data were collected from the hospital archives after approval of the Ethics Committee.

\section{CONFLICT OF INTEREST:}

The authors declared no conflict of interest.

\section{AUTHORS' CONTRIBUTION:}

HY: Substantial contributions to the conception or design of the work; or the acquisition, analysis, or interpretation of data for the work.

GD: Drafting the work or revising it critically for important intellectual content.

YS: Contributed to collection and analysis of data.

AY: Collected and analysed data, literature review and discussion.

\section{REFERENCES}

1. Bray F, Ferlay J, Soerjomataram I, Siegel RL, Torre, LA, Jemal A. Global cancer statistics 2018: Globocan estimates of incidence and mortality worldwide for 36 cancers in 185 countries. CA Cancer J Clin 2018; 68(6):p.394-424. DOI: 10.3322/caac.21492.

2. Schulz C, Kullmann F, Kunzmann V, Fuchs M, Geissler M, Vehling-Kaiser $U$, et al, Neoflot: Multicenter phase II study of perioperative chemotherapy in resectable adenocarcinoma of the gastroesophageal junction or gastric adenocarcinoma-Very good response predominantly in patients with intestinal type tumors. Int J Cancer 2015; 137(3): p.678-85. DOI: 10.1002/ijc.29403.

3. Mandard AM, Dalibard F, Mandard JC, Marnay J, Henry-Amar M, Petiot JF. et al. Pathologic assessment of tumor regression after preoperative chemoradiotherapy of esophageal carcinoma. Clinicopathologic correlations. Cancer 1994; 73(11):p.2680-6. DOI: 10.1002/1097-0142 (19940601)73:11<2680::aid-cncr2820731105> 3.0.c0;2-c.

4. Scholzen T, Gerdes J. The Ki-67 protein: from the known and the unknown. J Cell Physiol, 2000; 182(3):p.311-22. DOI: 10.1002/(SICI)1097-4652(200003)182:3<311::AIDJCP1>3.0.CO;2-9

5. Luo G, Hu Y, Zhang Z, Wang P, Luo Z, Lin J, et al. Clinicopathologic significance and prognostic value of Ki-67 expression in patients with gastric cancer: A meta-analysis. Oncotarget 2017; 8(30): p. 50273-50283. DOI: 10.18632/ oncotarget.17305.

6. Kim EY, Lee JW, Yoo HM, Park CH, Song KY. The platelet-tolymphocyte ratio versus neutrophil-to-lymphocyte ratio: Which is better as a prognostic factor in gastric cancer? Ann Surg Oncol 2015; 22(13):p.4363-70. DOI: 10.1245/ s10434-015-4518-z.

7. Gu X, Gao XS, Cui M, Mu Xie, Chuan Peng, Yun Bai, et al. Clinicopathological and prognostic significance of platelet to lymphocyte ratio in patients with gastric cancer. Oncotarget 2016; 7(31):p. 49878-49887. DOI: 10.18632/ oncotarget. 10490.

8. Quigley DA, Kristensen V. Predicting prognosis and therapeutic response from interactions between lymphocytes and tumor cells. Mol Oncol 2015; 9(10): p.2054-62. DOI: 10.1016/j.molonc.2015.10.003.

9. Durhan A, Senlikci A, Kosmaz K, Erguder E, Mercan U, Suleyman M. An evaluation of the effect of preoperative inflammation-based factors on survival in gastric cancer patients. J Coll Physicians Surg Pak 2021; 31(03):282-287. DOI: 10.29271/jcpsp.2021.03.282.

10. Kwon HC, Kim SH, Oh SY, Suee Lee, Ji Hyun Lee, Hong-Jo Choi, et al. Clinical significance of preoperative neutrophillymphocyte versus platelet-lymphocyte ratio in patients with operable colorectal cancer. Biomarkers 2012; 17(3):p. 216-22. DOI: 10.3109/1354750X.2012.656705.

11. Langsenlehner T, Pichler M, Thurner EM, Krenn-Pilko S, Stojakovic T, Gerger A, et al. Evaluation of the platelet-tolymphocyte ratio as a prognostic indicator in a European cohort of patients with prostate cancer treated with radiotherapy. Urol Oncol 2015; 33(5): p.201.e9-16. DOI: 10.1016/j.urolonc.2015.02.002.

12. Quéro L, Guillerm S, Hennequin C. Neoadjuvant or adjuvant therapy for gastric cancer. World J Gastrointest Oncol 2015; 7(8): p.102-10. DOI: 10.4251/wjgo.v7.i8.102.

13. Li R, Heydon K, Hammond ME, David J Grignon, Mack Roach 3rd, Harvey B Wolkov, et al. Ki-67 staining index predicts distant metastasis and survival in locally advanced prostate cancer treated with radiotherapy: an analysis of patients in radiation therapy oncology group protocol 86-10. Clin Cancer Res 2004; 10(12 Pt 1): p. 4118-24. DOI: 10.1158/ 1078-0432.CCR-1052-03.

14. Inari H, Suganuma N, Kawachi K, Yoshida T, Yamanaka T, Nakamura $Y$, et al. Clinicopathological and prognostic significance of $\mathrm{Ki}-67$ immunohistochemical expression of distant metastatic lesions in patients with metastatic breast cancer. Breast Cancer 2017; 24(6):p.748-755. DOI: 10. 1007/s12282-017-0774-z.

15. Li W, Zhang G, Wang HL, Wang L. Analysis of expression of cyclin E, p27kip1 and Ki67 protein in colorectal cancer tissues and its value for diagnosis, treatment and prognosis of disease. Eur Rev Med Pharmacol Sci 2016; 20(23): p.4874-4879. PMID: 27981549.

16. Templeton AJ, Ace O, McNamara MG, Al-Mubarak M, VeraBadillo FE, Hermanns T, et al. Prognostic role of platelet to lymphocyte ratio in solid tumors: A systematic review and meta-analysis. Cancer Epidemiol Biomarkers Prev 2014; 23(7):p.1204-12. DOI: 10.1158/1055-9965.EPI-14-0146.

17. Zhao QT, Yuan Z, Zhang H, Xiao-Peng Z, Hui-En W, Zhi-Kang $W$, et al. Prognostic role of platelet to lymphocyte ratio in non-small cell lung cancers: A meta-analysis including 3,720 patients. Int J Cancer 2016; 139(1): p.164-70. DOI: 10.1002/ ijc.30060.

18. Lu C, Gao P, Yang Y, Chen X, Wang L, Yu D, et al. Prognostic evaluation of platelet to Iymphocyte ratio in patients with colorectal cancer. Oncotarget 2017; 8(49):p. 86287-86295. DOI: 10.18632/oncotarget.21141.

19. Sun Z, Zhu RJ, Yang GF, Yan Li. Neoadjuvant chemotherapy with FOLFOX4 regimen to treat advanced gastric cancer improves survival without increasing adverse events: A retrospective cohort study from a Chinese center. ScientificWorldJournal 2014; p.418694. 
DOI: $10.1155 / 2014 / 418694$.

20. Wu A, Jia Y, Dong B, Tang L, Liu Y, Hong Du, et al. Apoptosis and $\mathrm{KI} 67$ index correlate with preoperative chemotherapy efficacy and better predict the survival of gastric cancer patients with combined therapy. Cancer Chemother Pharmacol 2014; 73(5):p.885-93. DOI: 10.1007/s00280014-2410-3.

21. Qu J, Qu X, The predictors of response to neoadjuvant chemotherapy in patients with locally advanced gastric cancer. Cancer Biomark 2016; 17(1):p.49-54. DOI: 10.3233/ CBM-160616.

22. Lian, L, Xia YY, Zhou C, Xiao-Ming S, Xiang-Li L, Shu-Guang $H$, et al. Application of platelet/lymphocyte and neutrophil/lymphocyte ratios in early diagnosis and prognostic prediction in patients with resectable gastric cancer. Cancer Biomark 2015; 15(6):p.899-907. DOI: 10.3233/ CBM-150534.
23. Wang F. Liu ZY, Xia YY, Chong Z, Xiao-Ming S, Xiang-L, et al. Changes in neutrophil/lymphocyte and platelet/ lymphocyte ratios after chemotherapy correlate with chemotherapy response and prediction of prognosis in patients with unresectable gastric cancer. Oncol Lett 2015; 10(6):p.3411-3418. DOI: 10.3892/ol.2015.3783.

24. Chen L, Hao Y, Cong X, Menghua Zou, Sen Li, Lihua Zhu, et al. Peripheral venous blood platelet-to-lymphocyte ratio (PLR) for predicting the survival of patients with gastric cancer treated with SOX or XELOX regimen neoadjuvant chemotherapy. Technol Cancer Res Treat 2019; 18:p.1533 033819829485. DOI: 10.1177/1533033819829485.

25. Gong W, Zhao L, Dong Z, Yu Dou, Yanguo Liu, Chao Ma, et al. After neoadjuvant chemotherapy platelet/lymphocyte ratios negatively correlate with prognosis in gastric cancer patients. J Clin Lab Anal 2018; 32(5):p.e22364. DOI: 10.1002/jcla.22364. 